\title{
HUME POR DELEUZE. EL EMPIRISMO COMO GÉNESIS DE LA SUBJETIVIDAD
}

\author{
Felipe Larrea Melgarejo ${ }^{1}$ \\ Universidad de Chile
}

Recibido: 11.05.2018 - Aceptado: 06.07.2018

\begin{abstract}
RESUMEN
En 1953 Deleuze publica su primer libro, llamado Empirisme et subjetivité. Essai sur la nature humain selon Hume. Deleuze señala en primer lugar una afirmación distinta con respecto al empirismo de Hume, es decir, señalar que el empirismo es definible a partir de una relación intrínseca con los sentidos, y que el conocimiento, en primera instancia, proviene de ellos. Lo que advertirá Deleuze es que el empirismo debe ser definido, antes que todo, en relación con el problema de la subjetividad. El empirismo constituirá una doctrina que plantea que la subjetividad no está dada, sino que, más bien, debe ser producida o constituida (a esto le llamaremos una génesis de la subjetividad). ¿De qué manera se produce la génesis? A través de la acción de ciertos principios que son extensivos a la naturaleza humana: la pasión y la asociación. El Hume de Deleuze versará sobre dicho movimiento, y le permitirá al autor francés elaborar un primer índice de su metafísica, o lo que llamará, unos años después, empirismo trascendental.
\end{abstract}

Palabras clave: Hume, empirismo, génesis, subjetividad, principios.

\begin{abstract}
In 1953 Deleuze published his first book, Empirisme et subjetivité. Essai sur la nature humain selon Hume. Deleuze points out in the first place a different statement regarding Hume's empiricism, namely to indicate that empiricism is definable in terms of an intrinsic relation with the senses, and that knowledge, in the first instance, comes from them. Deleuze will remark that empiricism must be defined, first of all, in relation to the problem of subjectivity. Empiricism will constitute a doctrine that states that subjectivity is not given, but rather must be produced or constituted (we will call this a "genesis of subjectivity"). In what way is the genesis produced? Through the action of certain principles that are extensive to human nature: passion and association. Deleuze's Hume will deal with this movement, and will allow the French author to elaborate a first index of his metaphysics, which he will call a few years later "transcendental empiricism".
\end{abstract}

Keywords: Hume, empiricism, genesis, subjectivity, principles.

\footnotetext{
${ }^{1}$ larrea.felipe@gmail.com
} 


\section{INTRODUCCIÓN}

Nos proponemos identificar qué significa empirismo en el contexto de la primera obra de Gilles Deleuze, y extraer ciertas consecuencias que tomarán mayor forma en los años posteriores a la publicación de Empirisme et subjetivité. ${ }^{1}$ El estudio que escribiera Deleuze sobre Hume en 1953 se nos presenta como el primer movimiento que el filósofo francés realiza con respecto a su metafísica, aunque sea más común, entre los comentadores de su obra, mantenerlo en una posición más marginal, no solo por los nueve años que lo separan de su segundo libro (Nietzsche et la philosophie [NP]) sino además por tener un aire temático un poco disímil y distante con el resto de su trabajo, pero, sobre todo, por poseer una terminología díscola en comparación a sus obras futuras. ${ }^{2}$ Dicho prejuicio se sostiene, mayormente, por la relevancia que conserva el concepto de subjetividad, en el sentido de que en sus obras posteriores se le ubicó - y el mismo Deleuze pareciera reafirmarlo- como un filósofo en contra de la noción de subjetividad y, por extensión, a la de sujeto. Cuestión que nos parece poco rigurosa en el acercamiento hacia su obra, ya que si bien nunca pensó al sujeto como sustancia, sí reformuló una aproximación a este problema, y es justamente en su lectura a Hume donde pareciera actualizar una discusión que en el siglo XX parecía superada. ${ }^{3}$

Ahora bien, creemos que existen dos vectores fundamentales de $E S$, que podríamos llamar programáticos, en donde se liga lo que llamaremos una génesis de la subjetividad, que será extensiva en otros lugares del pensamiento deleuzeano. En primer lugar, un vector metodológico, si lo pudiéramos llamar así, que es la impronta más de historiador de la filosofía que Deleuze en su primera época encarnó; y en segundo lugar, un lineamiento de objetivos temáticos, como conceptuales, en los cuales los primeros trabajos de Deleuze preparan su "propio" pensamiento. A saber, ES, constituye una obra - como dirá en un prólogo escrito en 1991 para su edición en lengua inglesa - que enuncia una premisa fundamental, resumida de la siguiente

\footnotetext{
${ }^{1}$ En adelante $E S$.

${ }^{2}$ Existe poca bibliografía de comentario sobre $E S$, más allá de algunos artículos determinados y acotados a este estudio, y en otros casos puesta en relación a sus obras futuras, sobre todo con respecto al vínculo con Bergson, y con ciertas aristas de Différence et répétition.

${ }^{3}$ En un pequeño texto de 1988, titulado "Réponse a une question sur le sujet", Deleuze señalará lo siguiente: "creemos que la noción de sujeto ha perdido mucho de su interés en beneficio de las singularidades pre-individuales y de las individuaciones impersonales. Pero, precisamente por ello, no basta oponer unos conceptos a otros para saber cuál de ellos es mejor, es preciso confrontar los campos problemáticos a los que responden para descubrir las fuerzas que están transformando los problemas y exigiendo la constitución de nuevos conceptos" (2003, 328).
} 
manera: "Hume anuncia a Kant" (Deleuze 2003, 341). Dicha cuestión, que no solo está expresada para lo que respecta al estudio de la obra de Hume, sino además con respecto al camino, o a cierto trazado de pensamiento, que lo llevará oblicuamente de Hume a Kant y de Kant a Hume. Este camino, por supuesto, está cruzado y plegado con otros nombres de la historia subterránea que Deleuze escribirá en los años 50 y 60 del siglo pasado (Bergson, Spinoza, Nietzsche, Lucrecio, etc.). Sin duda, $E S$, antes que dicha relación con estos otros nombres, plantea una lectura proto-kantiana de Hume que trataremos de puntualizar. ${ }^{4}$ El segundo vector tendrá relación, como decíamos, con ciertos objetivos temáticos al interior del pensamiento deleuzeano, lo podríamos enmarcar de la siguiente manera: el esbozo de cierto principio de la inmanencia, al plegar lo pasional con lo social; y, del cómo la imaginación no constituye una facultad sino más bien se esgrime como lo dado. Estas dos zonas, finalmente, se condicen con lo que entenderá con respecto a la génesis de la naturaleza humana, o la subjetividad, a partir de sus principios, que no es sino el corazón mismo del empirismo en un sentido superior.

\section{EL EMPIRISMO COMO PSICOLOGÍA DE LAS AFECCIONES DEL ESPÍRITU}

Deleuze acentúa, desde las primeras páginas de $E S$, cierta elección acometida por Hume que es fundamental para lo que se afirmará en torno al empirismo. El problema tiene relación con redefinir el estatuto de la psicología, afirmada como ciencia o saber del espíritu. Para Deleuze, Hume habría descartado ser meramente un psicólogo, apostando en su elección por una psicología de las afecciones del espíritu, en vez de una psicología del espíritu. Vemos que el desplazamiento preciso que subraya Deleuze, tiene relación con el papel fundamental de la afección: solo del espíritu no es posible ninguna ciencia, es necesario pues, afirmar la potencia de las afecciones. ${ }^{5}$ A partir de

\footnotetext{
${ }^{4}$ Como advierte Patricia De Martelaere, de cierta manera en la lectura (singular) de Deleuze sobre Hume, pareciera actualizar un léxico exterior al propio Hume, en el sentido de presentar al pensador escocés dentro de cierta problemática "contemporánea", y por cierto, con una marcada inspiración kantiana. Así los conceptos como "sujeto", "origen" o "constitución" dan cuenta de una actualización bastante marcada dentro del estudio que ofrece Deleuze, y no están expuestas como parte de un debate anacrónico (1984, 224-5).

${ }^{5}$ En un trabajo que tendremos en la mira en gran parte de este artículo, Ernesto Feuerhake advertirá la relevancia de la cuestión de la afección en las primeras páginas de $E S$, donde señala que si las dos formas de la afección, son lo social y lo pasional, esto se debe mayormente a la elección que ha acometido Hume con respecto a no ser solo un psicólogo, sino un moralista y un sociólogo: "Un moralista, por la afección pasional; un sociólogo, por la afección social (...) Estas dos formas de la afección, agrega Deleuze, "se implican", y al implicarse aseguran "la unidad del objeto de una ciencia auténtica". La ciencia del hombre, la "psicología de las afecciones", tendrá su objeto "asegurado" debido a la mutua implicación de lo pasional y lo social en cuanto que formas de la afección. Lo cual quiere decir que esa mutua implicación asegura para un objeto una constancia y una universalidad que lo hacen susceptible de una "ciencia auténtica", "verdadera ciencia del hombre", ya no falsa ciencia como la psicología sin más" (Feuerhake 2014, 71-72). Del cómo ocurre esta implicancia es parte de los objetivos que debemos desarrollar.
} 
esta distancia, que se emprende como una elección decisiva, se plantea la tarea del proyecto empirista levantado por Hume, que sintetiza Deleuze en esta pregunta, que de cierta forma constituye una de las preguntas fundamentales para la modernidad: “cómo es que el espíritu deviene sujeto?” (Deleuze 1953, 3). Es en este sentido que la lectura que Deleuze enmarca en $E S$, atiende al propósito de Hume con respecto a realizar una verdadera ciencia, que tiene la tarea de advertir ciertas reglas o principios de la naturaleza humana. Esta cuestión fundamental para el proyecto de Hume es trazada a medida que se va planteando una re significación de la experiencia, y con ello, otra forma de sugerir la producción de la subjetividad, o más bien, de delimitarla precisamente como una producción específica.

De mano de lo anterior, Deleuze observará que tradicionalmente el empirismo fue mal comprendido por la historia del pensamiento, situándolo en una oposición simple contra el racionalismo, como si su irrupción se relacionara con una inversión en una zona que no necesariamente es lo fundamental para el empirismo, a saber, el conocimiento (Deleuze 1953, 118). Para Deleuze, el empirismo desde Hume no podrá definirse como una doctrina que postula que el conocimiento deriva de la experiencia sensible, sino que, más bien, el empirismo apuesta por pensar cierta génesis de la naturaleza humana. Dicha cuestión es enfatizada por Deleuze cuando señala que el empirismo no trata de resolver un "origen del espíritu" ya que el espíritu no será para Hume un lugar constituyente, sino que, por el contrario, advertirá que el sujeto es producto de una génesis que ocurre en el espíritu. Sin embargo, antes de afirmar esto, Deleuze puntualiza detenidamente como es que Hume, en el Tratado sobre la naturaleza humana, sostiene que el espíritu es afectado bajo dos formas que se implican, y que en principio, parecieran remitir a esferas que no se relacionan: lo pasional y lo social (Deleuze 1953, 1). La única forma de elaborar una verdadera ciencia de la naturaleza humana es a partir de esta implicancia, o como estos dos principios entran en juego. ${ }^{6}$

\footnotetext{
6 "En resumen, la elección del psicólogo se podría expresar extravagantemente así: ser un moralista, un sociólogo y un historiador antes que ser un psicólogo, para ser un psicólogo" (2). En la primera edición al castellano de Empirismo y subjetividad, realizada por Editorial Granica en 1977, el subtítulo elegido fue el siguiente: "Las bases filosóficas del Anti-Edipo". Independiente de lo estrictamente publicitario de ese subtitulo, los editores apuntaban a una diferencia que ya se marcaba en ese entonces dentro del corpus deleuzeano. Una distinción que se basa en que existirían obras - las previas al encuentro con Guattari - que son moduladas como más "filosóficas" que otras. Sin duda que $E S$ constituye un libro que adelanta tópicos y vectores que serán desarrollados, pero también radicalizados, en esa primera obra junto a Guattari. Pero creemos que ES significa para el pensamiento de Deleuze una suerte de instalación de gran parte de los problemas que trabajará en sus obras posteriores, y que quizás se coronan en el L'Anti-OEdipe (en adelante, $A O E$ ). Ahora bien, con respecto a la cita recién expuesta, podríamos pensar que cuando Deleuze y Guattari hablan de una psiquiatría materialista o en términos generales del esquizoanálisis, es de una u otra manera Hume el autor que está referenciado implícitamente. La crítica central a Freud, y al psicoanálisis, podría ser refrendada bajo la misma óptica de la elección que habría hecho Hume con respecto a la psicología. A saber, el psicoanálisis hace del inconsciente un teatro, una mera representación, en donde Edipo y la triangulación familiar, explican o metaforizan el inconsciente. El desplaza-
} 
Para poder alzar esta ciencia, antes que todo, su propia condición, es que el espíritu pueda ser afectado. Es en este sentido que el espíritu no es una naturaleza particular, ya que para que ella devenga objeto de conocimiento, como decíamos, debe ser presa de una afección. De este modo, que desde un libro como $E S$, para Deleuze el conocimiento no es un ámbito de la naturaleza humana que deba ser descubierto, como si el objeto de él estuviera dado. Más bien, el conocimiento para que se haga real debe provenir de cierta afección, de cierta violencia, de cierto encuentro: sin afección no existe posibilidad de ciencia de la naturaleza humana.

Ahora bien, qué es el espíritu no es algo que la filosofía de Hume sistematiza, es que de cierta manera esta concepción del espíritu está determinada por la manera de trabajar filosóficamente del empirismo de Hume: el espíritu no es causa ni agente de producción, lo importante de establecer más bien, es cómo el espíritu deviene naturaleza humana, o en otras palabras, cómo deviene sujeto. Es lo que decíamos a partir de que no existe para el empirismo un problema con respecto al "origen" del espíritu, el problema más bien comienza en cómo establecer $s u$ devenir. Por esto, es que el espíritu necesita ser afectado para que aquello se dé como una forma a ser conocida, para que se convierta en cierto material, para que exista, en definitiva, conocimiento. Lo que fuerza proviene de un afuera de la "naturaleza humana". Dicho afuera es enunciado como la acción de ciertos principios, que cumplen el papel, en cierto momento, de fijar al espíritu y de afectarlo. Este efecto que provocan los principios es aquello que, a ciencia cierta, se llamará génesis de la subjetividad, cuestión que por ejemplo para Gonzalo Montenegro será primordial con respecto al hábito, y en esta relación problemática se esgrime el verdadero núcleo del empirismo (Montenegro 2013, 6-7). Como advertíamos en la nota anterior, por esta razón es que el problema del hábito abre una vía específica y particular de la lectura de Hume, que debe a nuestro parecer ser cotejado de manera independiente, pues abre diversas vías de investigación, y por cierto que también otros textos del mismo Deleuze. Ahora bien, por esto nuestra necesidad de reafirmar que la idea de una génesis de la subjetividad en $E S$ constituye un desplazamiento específico, que es el que nos interesa demarcar por ahora.

miento de Deleuze y Guattari consistía en pensar el inconsciente como una fábrica, y su proceso, como un verdadero proceso de producción, social y político. En otros términos, en el inconsciente se daba cita una implicación entre un principio social y pasional, político y afectivo, que de cierta manera constituye la comprensión del deseo — siendo quizás la tesis más fuerte de ese libro - como principio inmanente (Deleuze — Guattari 1972, 10-1). Sin embargo, la cercanía más notoria entre $E S$ y $A O E$, que es con respecto al problema de la síntesis, y en particular el problema que concierne al hábito como principio, será enunciada como una síntesis pasiva, no solo en Différence et répétition (en adelante, $D R$ ), sino también en $A O E$. La síntesis pasiva constituye el terreno de lo que ahí se llamarán las síntesis del inconsciente (Deleuze - Guattari 1972, 34; 388-9), sin embargo es un problema que deberíamos cotejar en otro lugar por su extensión y complejidad. 


\section{LA TRIPLE IDENTIDAD (ESPÍRITU - IDEA - IMAGINACIÓN)}

La pregunta que aparece en las primeras páginas de la lectura que Deleuze ofrece de Hume, es cómo aquello que no es origen sino una suerte de medio, como es el espíritu, se encuentra dado. Una vía bajo la cual Deleuze intenta despejar este problema la manifiesta cuando señala que "lo dado ya no está dado a un sujeto; el sujeto se constituye en lo dado" (Deleuze 1953, 92). Esta fórmula querría decir que "lo dado" en Hume no constituye una sustancia, más bien se manifiesta como un "flujo de lo sensible", compuesto por "movimiento" e "imágenes". A esta suerte de colección, de "pieza sin teatro", es lo que Hume llama "espíritu", pero al mismo tiempo, "imaginación". La insistencia que se debe precisar, es que lo dado no está sujetado, ya que tiende en extremo al movimiento, al cambio o la alteración, sin posibilidad de fijarlo para el conocimiento. Como se verá, Deleuze trama una concepción distinta de aquello con lo cual el empirismo trabaja, en tanto doctrina, ya no es la experiencia en primer término, pues la experiencia no es algo que está cualificado para un sujeto, la experiencia en este sentido se debe producir. Sin embargo, existe un nivel en las precisiones que realiza Hume, en donde se puede ubicar la experiencia, ese lugar no es otro que este flujo de lo sensible que es la imaginación o el espíritu.

Sin cesar Hume afirma la identidad del espíritu, la imaginación y la idea. EI espíritu no es naturaleza; no tiene una naturaleza. Es idéntico a la idea en el espíritu. La idea es lo dado, tal como es dado; es la experiencia. EI espíritu es dado. Es una colección de ideas, no alcanza a ser un sistema. Y la cuestión precedente podría expresarse así: ¿cómo una colección deviene un sistema? La colección de ideas se llama imaginación, en la medida en que designa, no una facultad, sino un conjunto, el conjunto de las cosas, en el más vago sentido de la palabra, que son lo que parecen: colección sin álbum, pieza sin teatro, o flujo de percepciones (Deleuze 1953, 3. Cursiva nuestra). ${ }^{7}$

De este modo, Hume afirma una triple identidad, entre el espíritu, la imaginación y la idea (la idea en el espíritu), para diseñar una comprensión de lo dado no naturalizado, bajo todo el sentido de lo que esa palabra señala, es decir, "el espíritu no es una naturaleza". Sin embargo, lo que podría ser sencillo en este nivel, adquiere una complejidad que solo en la medida en

\footnotetext{
${ }^{7}$ Las traducciones de los textos originales de Deleuze son mías, sin embargo, son cotejadas con las versiones disponibles en castellano. Por esto mismo, serán referenciadas en la bibliografía general del artículo.
} 
que va avanzando el trabajo de Deleuze en torno a Hume se podrá comprender en todo su alcance. No existe distinción real entre la idea y el espíritu, en tanto la idea está dada, tal cual como el espíritu. Lo singular, que Hume promueve, es que la idea, en este sentido constituye la experiencia tal cual como se encuentra dada. Pero debemos atender a que la experiencia, para Hume, no es una cuestión meramente sensible, o que está dada sin más para un sujeto, porque todo se formula y se remarca de ese modo, a saber, que el sujeto tendrá que ser producido, tendrá que germinar. Acá se encuentra, sin lugar a dudas, el primer movimiento que podrá definir el empirismo que Deleuze lee en Hume, que como iremos advirtiendo, se desprende del diseño de su propio empirismo, como también, su propia metafísica, quizás reversos de un mismo horizonte. Ahora bien, si el espíritu debe devenir sujeto, del mismo modo la imaginación debe devenir facultad, ambas transformaciones son imagen de un mismo proceso, o en términos más precisos, de una misma génesis.

En particular, el problema de la imaginación, reside en que si se entabla como lo dado es a partir de su definición como "colección de ideas", no alcanzando a constituirse como una facultad. Dicha cuestión quiere decir que no es algo que le pertenece a un sujeto, más bien, la idea de imaginación de Hume tiene relación con un "flujo de percepciones", que ante todo tiene como cualidad al movimiento, al cambio, por esto mismo es que no constituye bajo ningún motivo una sustancia. De allí se desprende que el objeto del empirismo es la imaginación y no la experiencia, ni los sentidos (Deleuze $1953,124)$. Trama que expresa, de manera bastante rigurosa, que el conocimiento no es el objeto primero del empirismo, no está en su horizonte. Del mismo modo que la experiencia no tiene un significado preciso en la obra de Hume, y tampoco es el destino del empirismo, al menos, no en un significado de experiencia aún atrapado bajo una metafísica de la sustancia o estrictamente racionalista. A Hume, según observa Deleuze, no valdría ubicarlo como un pensador que pretende ir a algún origen, ni del conocimiento, ni del entendimiento, sino que, más bien, a partir de ciertos efectos va a un lugar previo a las constituciones, tanto de la imaginación como del sujeto. De ahí su pregunta, cómo es que aquello devino, cómo es la génesis del sujeto, cómo es la génesis de la imaginación en tanto facultad. Para decirlo de manera fuerte: el espíritu en Hume no es otra cosa que sus propios contenidos. Esto significa que se confunde con ellos, ya que estos contenidos son las ideas, y es en este sentido que enunciamos a la idea como idea en el espíritu, en tanto es sus contenidos. A su vez también la siguiente fórmula: la idea está en la imaginación, así como la imaginación es definida, precisamente, como una "colección de ideas". La inmanencia descriptiva de Hume es lo que a Deleuze permite enunciar que lo dado no es homologable a ningún tipo de sustancia, ni tampoco a ningún agente causal que la produzca: "nada se hace por la imaginación [sino que] todo se hace en la imaginación” (Deleuze 1953, 3). 
La distinción que opera Deleuze está al servicio de delimitar la imaginación, no es una noción, o más precisamente un lugar, desde donde se produzca algo, ni las ideas, ni las percepciones. Tampoco se puede simplemente señalar que la imaginación es un lugar donde habiten las ideas, puesto que si el movimiento es aquello singular que se promueve desde la imaginación, las ideas no se encuentran fijas, por el contrario, son móviles, y por esta razón la imaginación debe fijarse para que de ahí se constituya realmente como un lugar en sí. Esa es la labor que realizará la afección, o el accionar de los principios de la naturaleza humana, ya que antes de esa sacudida, la imaginación solo es movimiento de las ideas, acción y reacción, lugar azaroso, desde el cual no puede germinar ningún tipo de saber, ni de conocimiento. Es en este sentido que Deleuze define a la imaginación como un determinable y no como una determinación determinante (Canavera 2012, 127). Si bien la imaginación posee una actividad, esta para Deleuze es inconstante, azarosa, carece de uniformidad, pues es fantasiosa y delirante. Es que se debe precisar que aquí, imaginación, espíritu e idea en el espíritu, se identifican con lo pasivamente dado. Lo dado es pura pasividad. Por otro lado, la imaginación no puede proveer los elementos para establecer una real ciencia, ya que no tiene determinación siendo exclusivamente determinabilidad. En otras palabras, lo que Deleuze está abriendo en las primeras páginas de $E S$ consiste en cierta cuestión fundamental que tiene relación con la necesidad de principios: el espíritu necesita de algunos principios para devenir naturaleza humana.

El fondo del espíritu es delirio, o, lo que quiere decir lo mismo desde otros puntos de vista, azar, indiferencia. Por sí misma, la imaginación no es una naturaleza, sino una fantasía. La constancia y la uniformidad no están en las ideas que tengo. Como tampoco en la forma en que las ideas son ligadas por la imaginación: es una vinculación que se efectúa al azar (Deleuze 1953).

Que Hume no haya definido a la imaginación como una facultad constituida, se debe al hecho de anteponerse a una simple psicología del espíritu, como señalábamos en un comienzo. La imaginación no produce, no es activa, lo que Hume se propone al decir de Deleuze, es que se debe fundamentar cómo es que la imaginación deviene facultad. Aun así la paradoja es que en la imaginación se producen las ideas, al menos, se ligan, tienen un vínculo. Sin embargo, la imaginación no es la que liga ni asocia, aunque ocurra en ella. De otra manera se dirá, y esto es lo clave para lo que significará el empirismo, es que si las ideas se asocian, es por un principio exterior a la misma imaginación. Este principio, Hume lo llama un principio de asociación que viene a determinar a la imaginación y a las ideas en el espíritu. De ahí la 
célebre sentencia de $E S$, con respecto a la exterioridad de las relaciones, pues estos principios son exteriores a las ideas que modifican, que alteran, es también por dicha cuestión que el empirismo de Hume es una suerte de fisicalismo (Deleuze1953, 63). ${ }^{8}$

\section{QUÉ SIGNIFICAN LOS PRINCIPIOS DE LA NATURALEZA HUMANA}

Antes de advertir que la producción de los principios tiene vínculo con un cierto afuera, habría que precisar la importancia decisiva que posee el empirismo que Hume promueve. Volviendo al pasaje que comentábamos un poco más arriba, a propósito de la triple identidad del espíritu con la imaginación y la idea (en el espíritu), se advertía allí que la idea se circunscribe como lo dado, y la idea constituye la experiencia en tanto está dada. Sin embargo, esta colección de ideas sin conexión, presas de un azar sin uniformidad, son afectadas por un principio que hará ir a la experiencia, más allá de sí, estableciéndose un carácter más singular, que finalmente será el verdadero objeto del empirismo, en un sentido superior. Señala Hume:

Como todas las ideas simples pueden ser separadas por la imaginación y unidas de nuevo en la forma que a ésta le plazca, nada sería más inexplicable que las operaciones de esta facultad si no estuviera guiada por algunos principios universales que la hacen, en cierto modo, conforme consigo misma en todo tiempo y lugar (Hume 2005, 54).

Para Hume, este principio es la asociación, que se esgrime como un verdadero principio de la naturaleza humana. La imaginación no puede ella misma asociar las ideas pues necesita de un principio que proviene desde fuera y que la estabiliza, la fija, produciendo su devenir facultad humana: "la asociación rebasa (dépasse) a la imaginación" (Deleuze 1953, 4). Es por este motivo que a la imaginación se le fija una naturaleza, que se constituye en

\footnotetext{
8 "Si hubiera que definir el todo, se lo definiría por la Relación. Pues la relación no es una propiedad de los objetos, sino que siempre es exterior a sus términos. Además es inseparable de lo abierto, y presenta una existencia espiritual o mental. Las relaciones no pertenecen a los objetos, sino al todo, a condición de no confundirlo con un conjunto cerrado de objetos" (Deleuze 1983, 20). Independiente que el problema de las relaciones no es algo que explícitamente está formulado en Bergson, sin embargo creemos que la concepción del Todo, que aparece en L'Image-Mouvement da luz sobre cierta definición que Deleuze trabaja en $E S$, en el sentido de pensar a las relaciones por fuera de sus términos, fuera de sus atributos. Las relaciones más bien son definibles a partir de un conjunto, de un Todo, que no está cerrado ni tampoco se da como dado, sino que se esgrime como lo abierto o aquello en donde se podría producir lo nuevo. En otras palabras, las relaciones de las partes de un Todo son lo que transforman o afectan, a la propia duración.
} 
un después, provocada por el principio de asociación, para que así las ideas se relacionen o se vinculen. Aunque se debe especificar que la asociación no es una cualidad inherente a las ideas, así como tampoco a la misma imaginación, es un principio que proviene de un encuentro, de un choque, que violenta (afecta) a la imaginación. Es una "regla de la imaginación no un producto" (Deleuze 1953, 5), pero Deleuze un par de párrafos antes, había ya deslizado cierta idea de la asociación, ésta se le aparecía puntualmente como el entendimiento (Deleuze 1953, 2). Ahora bien, lo importante de delimitar, es que la asociación en tanto principio "afecta" a la imaginación (siendo otra cosa de ella) desde afuera, haciendo devenir a la imaginación una facultad, y al espíritu en naturaleza humana. Todo esto es profundamente significativo para aquello que decíamos en un comienzo, en la definición provisoria del empirismo que Deleuze comienza a buscar en Hume, es decir, el empirismo puede ser definido a partir de cierta génesis de la subjetividad.

Estamos por cierto en el centro de esa cuestión, al menos bordeando su espesor, que tiene total relación con este principio de la asociación de ideas en la imaginación: sin asociación no se rebasa al espíritu, y por ende, no existe génesis de la subjetividad. Lo pertinente de subrayar es que la asociación también tiene unos principios que la constituyen: la contigüidad, la semejanza y la causalidad. A partir de estos se rebasa lo dado, constituyéndose la subjetividad. Sin embargo, luego de constituirse el sujeto, en este "más allá" de lo dado, él debe creer, afirmar la experiencia o la existencia de algún modo. Deleuze, de este modo, enuncia cierto privilegio que tendría la causalidad, en el sentido de que "ella sola puede hacernos afirmar la existencia, hacernos creer, porque le confiere a la idea del objeto una solidez" (Deleuze $1953,5)$ que al parecer no tendrá con solo la contigüidad o la semejanza. En otros términos: sin causalidad no existe "mundo", sin este principio la asociación solo vincularía las ideas sin síntesis, quedando en meras impresiones e ideas. Lo que quiere decir, en este mismo punto de la lectura deleuzeana, es que la naturaleza humana "en sus principios va más allá del espíritu que nada en el espíritu va más allá de la naturaleza humana; nada es trascendental" (Deleuze 1953, 5). Nuevamente la precisión importante: el espíritu no es determinante, no determina a la naturaleza humana ¿Qué es lo que la determina? Pues bien, ella misma ocupa el lugar de un determinante que determina en la imaginación, en el espíritu, la asociación de ideas y con ello el entendimiento.

En este momento podríamos al menos prever una luz sobre la subjetividad: ella germina solo gracias a la afección provocada por unos principios que rebasan al espíritu y lo fijan. Nuevamente señalar que a Hume, no le interesa ir a las causas que originan estos principios, ni tampoco, como se habrá visto, reflexionar sobre una materia prima o agente que produce la subjetividad. Los principios son matizados solo en sus efectos, pues su acción 
es de por sí un efecto, y este es la producción de la subjetividad o la naturaleza humana. De esta manera, la paradoja que advierte Deleuze es la siguiente: ¿cómo es que el espíritu, o en el caso de la imaginación, deviene una naturaleza particular (del espíritu a naturaleza humana o de la imaginación a facultad) sin poseer en sí mismo la razón o el principio de ese devenir? “Por qué la naturaleza humana es la imaginación reglada más bien que la regla captada en su poder activo?” (Deleuze 1953, 5). En otros términos: ¿cómo se puede definir la actividad de un sujeto que se está constituyendo como pasividad? Nos adentramos, de esta forma, en un asunto crucial para el empirismo deleuzeano, es el asunto de la pasividad, que acá en el estudio sobre Hume pareciera tener un rol fundamental, pero no por eso explícitamente delimitado, más bien, es un problema que se trabaja bajo otros medios, pero cuando Deleuze retome a Hume, en $D R$, aparecerá en toda su dimensión problemática. ${ }^{9}$

Por ahora, la cuestión se plantea en cómo pensar realmente esta génesis de la subjetividad a partir de un conjunto, como es la imaginación, que no es agente productor sino más bien se encuentra regida por la pasividad. Deleuze pareciera sortear esta dificultad remarcando muy fehacientemente que el empirismo es una doctrina que escruta efectos, no busca causas: "la causa no puede ser conocida; no hay una causa de los principios, un origen de su poder. Lo original es su efecto sobre la imaginación" (Deleuze 1953, 6). Es que la acción de los principios siempre proviene de un afuera radical, las relaciones que se comienzan a asociar en la imaginación, es decir, en cómo estas ideas en el espíritu se vinculan, opera de manera gradual a como germina la subjetividad. También podríamos decirlo todo en otros términos: "el sujeto es la instancia” (Deleuze 1953, 109) bajo la cual se organiza la colección de ideas, sin embargo, el sujeto es exterior a las mismas ideas. Según Deleuze, la fórmula que se debe aplicar al empirismo, a partir de la génesis de la subjetividad, es que "las relaciones son exteriores a las ideas" (Deleuze 1953, 108), de James a Russell, pasando por Hume, es la verdadera declaración del empirismo. Retomaremos más adelante qué significa realmente que los principios no sean origen por sí mismos, como si estos principios del empirismo estuvieran lo suficientemente alejados tanto de la razón suficiente como de cualquier lógica del arjé. Por otro lado, estos principios provocan un devenir, y el devenir no tiene una razón determinada, solo es producto de una afección, de un golpe, o de un encuentro.

\footnotetext{
${ }^{9}$ La imaginación en el contexto de $D R$ estará definida como una "placa sensible" (Deleuze 1968, 96), sin embargo, su mayor característica es la pasividad, y porque en ella se produce una síntesis de índole pasiva.
} 


\section{EL REBASAMIENTO DE LA EXPERIENCIA}

Lo que Deleuze lee en Hume, como decíamos al principio con respecto a su proyecto, tiene relación con que para estudiar científicamente a la naturaleza humana, se la debe estudiar en el efecto que se produce en el espíritu. En otras palabras, la naturaleza humana no es originaria, más bien, aparece como efecto. Ahora, si volvemos a lo que Deleuze proyecta en las primeras páginas de $E S$, podríamos aducir que el empirismo de Hume, en tanto psicología de las afecciones del espíritu, se formula a partir de los principios de la naturaleza humana. Sin embargo, el espíritu, por sí mismo, es fundamental para esta ciencia, ya que en él se cualifica la naturaleza humana a partir de esos principios. En otras palabras, el espíritu es activado, es presa de una tendencia, singular, con la cual se puede decir que, efectivamente, ha devenido sujeto. Ahora bien, sin esta tendencia, como habíamos advertido, nos quedamos en el nivel de una psicología del espíritu que Deleuze grafica como una psicología de la idea, en el sentido que Hume piensa a la idea, a saber, como un elemento mínimo e indivisible, un átomo que ocupará una suerte de segunda dimensión con respecto al sistema del entendimiento. Es por esta razón que esta psicología del espíritu es otro nombre para el atomismo, tantas veces adjudicado al pensamiento de Hume. En cambio, el desplazamiento operado por Deleuze, es la de concebir al proyecto humeano a partir de una psicología de las afecciones del espíritu, que en términos amplios guarda relación con una tendencia introducida en el espíritu, en el sentido de que el espíritu ha devenido una naturaleza particular; por otro lado, en la imaginación las ideas se han comenzado a asociar, a partir del principio de la asociación. En otras palabras, esta psicología de las afecciones del espíritu, constituye otro nombre para el asociacionismo, precisión importante, ya que "confundir asociacionismo y atomismo es un extraño contrasentido" (Deleuze $1953,9)$. Sin embargo, el espíritu, como preocupación fundamental hasta este punto del recorrido que llevamos, podría abrir ciertas suspicacias con respecto a qué tan empirista es el problema de Hume. Deleuze parece presentir esa desconfianza, ya que en cierto momento interrumpe su propio desarrollo del problema para instarlo todo en otro plano, y señalar que "el problema de Hume concierne exclusivamente al hecho; es empírico. Quid facti?" (Deleuze 1953, 11).

¿Cuál es el hecho del conocimiento? La trascendencia o el rebasamiento (le dépassement); afirmo más de lo que yo sé, y mi juicio rebasa (dépasse) la idea. En otros términos: yo soy un sujeto. Digo: Cesar está muerto, y el sol saldrá mañana, Roma existe, yo hablo en general y creo, establezco relaciones, es un hecho, una práctica (Deleuze 1953, 11). 
Diez años después, en el inicio del primer capítulo de La philosophie critique de Kant (en adelante PCK), Deleuze retomará a Hume con el mismo ejemplo, para comenzar a definir lo que significaría lo a priori en el contexto de su estudio sobre Kant. El hecho del conocimiento estará delimitado bajo la figura de la necesidad y lo universal, cuestión expuesta en oposición a la experiencia, pues ella no nos da nada necesario ni universal. Se intuye, evidentemente, el tono kantiano al cual Deleuze apuesta para presentar y plantear el problema de lo trascendental. De este modo, la experiencia será aquello que se opone al ámbito de lo trascendental, o planteado en otros términos, existe un ámbito que condicionará a la experiencia. Estamos en presencia de una clave de lectura ya que si Deleuze ha señalado que el empirismo de Hume no versa, en primera instancia, sobre un concepto vulgar de experiencia, en donde el sujeto aún no se ha constituido, dicha cuestión es resultado de una apreciación resistente a pensar la experiencia como si estuviera dada en una relación de exterioridad con respecto al sujeto. Desde el léxico propuesto por Deleuze, en boca de Hume, se hablará de una noción de experiencia no cualificada por un principio de la naturaleza humana. Lo que pareciera estar de fondo acá, es que el empirismo no responde a una doctrina que se oponga totalmente a ninguna forma de lo a priori. Ahora bien, este es el horizonte de Hume, pero también el de Kant, de hecho es advertido precisamente por Deleuze, de la influencia de Hume en Kant, al menos en este punto (Deleuze 1963, 19). Sin embargo, es un punto de un encuentro para el propio pensamiento de Deleuze, que le servirá para postular que la experiencia no es lo radicalmente dado. La experiencia, más bien, debe ser parte de una singular germinación, ya que no constituye el objeto primero del empirismo, porque el empirismo (en un sentido superior pero también trascendental, entendido esto último deleuzeanamente) tendrá que ver siempre con lo genético más que con aquello simplemente dado. Es que lo dado se debe rebasar, lo dado, en tanto experiencia, bajo la acción de ciertos principios será "superado" para que de ello surja cierta subjetividad empírica.

Rebasamos o excedemos (dépassement) lo que no es dado en la experiencia gracias a principios que nos son propios, a principios necesariamente subjetivos. Lo dado no puede fundar la operación por la cual rebasamos lo dado. Sin embargo, no basta con que tengamos principios; también es preciso que tengamos ocasión de ejercerlos (Deleuze 1963, 19).

La cercanía entre Kant y Hume es manifiesta, al menos desde la inflexión que Deleuze está provocando, que como decíamos, no es solo que la experiencia se opone a lo dado para el sujeto, sino que también está en juego el 
papel primordial que tienen los principios. Remarca, Deleuze, que estos principios en Kant son necesariamente subjetivos. La sutileza de esta lectura no recae solo en lo que en $P C K$ advertirá inmediatamente sobre la diferencia que habría en Kant con respecto a Hume, es decir, de que finalmente a Kant se le imponía el Quid iuris más que el Quid facti. ${ }^{10}$ Esto último es a lo que Hume le compete, en tanto su problema es empírico; lo de Kant, evidentemente, pasa por establecer las coordenadas de lo trascendental. Se notará que en el estudio sobre Hume, Deleuze es bastante reacio a enmarcar o enmarcarse dentro de una perspectiva trascendental, de hecho, la soslaya. ${ }^{11} \mathrm{Sin}$ embargo, esta suerte de punto de partida que en $P C K$ es patente, en $E S$ pareciera estar en el centro de la configuración del empirismo humeano. Si bien plantea Deleuze que el problema del conocimiento es el hecho cognoscitivo, al desarrollarlo pareciera instalar una contradicción o una doble vía, que lo acerca al empirismo vulgar pero al mismo tiempo se encamina a una lectura proto-trascendental. Por eso es curioso que Deleuze deniegue lo trascendental, que está elucubrando en estas páginas de $E S$, y que se comenzará asomar en los artículos dedicados a Bergson a mediados de los años cincuenta.

Ahora, lo que debemos remarcar es que se abren dos vectores de lo dado y de la experiencia, ambos al decir de Deleuze son inversos, pues en un sentido hablamos de la experiencia como la idea que está en el espíritu, sin cualificación ni determinación; por otro lado, la experiencia del rebasamiento también se da, se da en el espíritu, rebasándolo a partir de la afección que provocan los principios haciendo real al sujeto. Dos nociones de experiencia, pero solo en la última es posible la subjetividad empírica, la naturaleza humana, y ésta es el objeto del empirismo, pero a su vez, se formula en una crítica radical a la psicología del espíritu o de la idea. La crítica que enarbola el empirismo lo será siempre de la idea no cualificada en aras de apostar

10 David Lapoujade ha advertido la primacía de la pregunta Quid Juris en la filosofía de Deleuze y que posee una marcada herencia kantiana, cuestión que al mismo tiempo define la impronta trascendental de su pensamiento: "sabemos que conlleva (el Quid Juris) una estricta repartición y un orden de las tareas; una vez establecido el hecho, hay que determinar con qué derecho procede, toda una "deducción" en el sentido jurídico, la famosa quaestio juris" (Lapoujade 2016, 27). Finalmente, la instalación de esta pregunta, crítica por naturaleza, tiene un alcance altamente político, con respecto a la pregunta sobre el territorio (“¿Cómo distribuir la tierra o el suelo? ¿A quién pertenece la tierra? ¿Quién decide de su distribución o de su reparto? ¿Y de qué tierra hablamos?” (Lapoujade 2016, 42).

11 "Vemos el fondo único del empirismo: es porque la naturaleza humana en sus principios rebasa (dépasse) al espíritu, nada en él rebasa (dépasse) a la naturaleza humana; nada es trascendental" (Deleuze 1953, 5). La distancia de Deleuze con respecto a lo trascendental en el estudio sobre Hume pasa por un desplazamiento que el empirismo produciría en relación a lo dado, pero en segundo lugar, aún en 1953 a Deleuze no se le aparecía, por llamarlo así, una dimensión de lo trascendental afirmativa, que formulará tímidamente en el ensayo "La conception de la différence chez Bergson" en 1956 pero que aparece de manera más contundente en "L’idée de genese dans l'esthétique de Kant" en 1963. Ahora, sin embargo, creemos que ya en el estudio sobre Hume existe un indicio importante de lo que en $D R$ (1968) llamará Empirismo Trascendental. Sin duda, esto que estamos desarrollamos ya da ciertas luces con respecto a aquello, independiente que terminológicamente excluya cualquier mención a lo trascendental. 
fuertemente por una psicología de la afección. En el contexto de ES, como hemos ya advertido, lo trascendental es objeto de una comprensión que, si bien, se alterará en las siguientes obras de Deleuze, el fondo de su distancia con lo trascendental en un sentido kantiano será básicamente el mismo. Para plantear el problema de la subjetividad, no se debe dar como supuesta la existencia a priori del sujeto, para Deleuze en estas páginas de $E S$ la crítica trascendental es constructiva en el sentido de formular la pregunta de “¿cómo algo puede darse a un sujeto?, ¿cómo el sujeto puede darse algo?” (Deleuze 1953, 92). Para la crítica en un sentido empirista, y en un sentido que se debe retener para la formulación del empirismo superior deleuzeano, el sujeto no se encuentra dado, no es por ningún motivo lo dado, sino que, más bien, la pregunta que plantea el empirismo es cómo el sujeto se constituye en lo dado (Deleuze 1953, 92). Deleuze observa que lo trascendental formula una lógica constructiva que debe tener su modelo en las matemáticas, en cambio el empirismo resalta una "física del espíritu y una lógica de las relaciones" (Deleuze 2002, 229). Dicha distancia que remarcamos, con respecto a lo dado, se nos presenta ya en esta primera obra de Deleuze bajo un tono similar a lo que más adelante desarrollará con respecto a su torsión de lo trascendental. La génesis de la subjetividad se constituye en lo dado, en su rebasamiento, tiene algo de lo dado, pero no se agota en él, no pre existe a la experiencia, no está presupuesto. Allí radica un primer vector, fuerte e importante, del empirismo que Deleuze ve en Hume.

\section{LA PASIÓN COMO PRINCIPIO}

Existen dos tipos de afecciones en el espíritu — las cuales habíamos mencionado en un comienzo - la pasión y la asociación (el entendimiento); si esta última vincula a las ideas en la imaginación, la pasión es la que entrega un sentido a esas relaciones. ¿Cómo es que ocurre?: la pasión, se refleja en la imaginación, al mismo tiempo que se extiende. Dicho movimiento es lo que constituye una regla general, es decir "la repercusión de la afección en el espíritu" (Deleuze 1953, 51). En este momento, Deleuze necesita distribuir las cosas en otro plano, o al menos, darle una vuelta al primer esquema que había advertido, el de la acción de los principios de la asociación en el espíritu, o en cómo la afección fijaba al espíritu dándole una naturaleza a la imaginación, produciéndose el devenir naturaleza humana. A esto se le llama un "efecto simple", o un primer nivel del problema de las afecciones en el espíritu. Por su cuenta, el segundo nivel, Deleuze le llama "el efecto complejo", que es lo que decíamos hace un momento: "la imaginación refleja (réfléchit) la afección; la afección resuena (retentit) en el espíritu” (Deleuze 1953, 52). Este resonar debe ser comprendido a la manera de Hume, es decir, como un sonido proveniente de un instrumento de percusión, pues el sonido, su vibración, va decayendo de forma gradual. La pasión es reflejada en la imaginación, se "amplía” dirá Deleuze, al "obligarla a rebasar (dépasser) los límites 
de su parcialidad" (Deleuze 2002, 234). Ahora, si las afecciones fijan al espíritu, dotándole una naturaleza y refleja estas afecciones, al mismo tiempo existe algo irreductible a la fijación provocada por las afecciones en la imaginación.

Dicha reserva, por llamarla de este modo, proviene de aquello que era la imaginación antes del golpe o del encuentro con los principios: sigue siendo, de una u otra manera, fantasía. En otros términos, existe un mínimo que se sustrae a la reflexión y que acontece con la pasión en la imaginación, por lo tanto, la pasión se levanta como aquello que dará cuenta de un lugar en donde prima lo singular, lo oscuro y lo profundo (Deleuze 1953, 115). Se advertirá que existe un problema en las locuciones que Deleuze utiliza para señalar aquella "reflexión" de la pasión en la imaginación, se encuentra "réfléchit", vocablo que se asemeja a un cierto espejeo, un reflejar; pero por otro lado se encuentra "réflexion", que se enuncia en el sentido de un pensamiento introspectivo, que se detiene de forma precisa en un objeto determinado. Ambos sentidos parecen plegarse, en el ejercicio que realiza el principio de la pasión al afectar a la imaginación, se refleja como en una reflexión. Ahora bien, decíamos que no era solo este efecto, sino que al mismo tiempo, en una suerte de identidad, la pasión se extendía en la imaginación, la ampliaba. En definitiva es la definición de la regla general para Hume, a saber, que la reflexión y la extensión se hace una en la imaginación (Deleuze 1953, 52, 3). Lo que ocurre, señala Deleuze, es que la pasión al reflejarse en la imaginación, en una imaginación ya naturalizada y fijada, se amplía, o dicho de manera más concluyente, resuena (retentit) en la imaginación. Sin embargo, se encuentra fijada no por la acción directa de la pasión, sino por el efecto del principio de la asociación: "es a través de los principios de la asociación que la pasión se refleja" (Deleuze 1953, 54). Se trata, de cierta manera, de una necesidad recíproca entre los dos principios, aunque pareciera que la asociación necesita de la pasión pues le otorga un sentido a la asociación de ideas en la imaginación. El modo de la asociación supone la existencia del reflejo de la pasión, "si las ideas se asocian, se asocian en función de una meta o de una intención, de una finalidad que únicamente la pasión puede conferir" (Deleuze 1953, 58), lo que en otras palabras significa que sin pasión no existiría necesidad de la asociación. ${ }^{12}$

\footnotetext{
12 Hume lo señala de la siguiente manera: "Como la naturaleza humana se compone de dos partes principales y necesarias en todas las acciones: las afecciones y el entendimiento, es cierto que los ciegos movimientos de las primeras, faltos de la dirección del segundo, incapacitan a los hombres para la sociedad. Y es licito que consideremos por separado los efectos resultantes de las operaciones separadas de estos dos componentes. La misma libertad que se permite a los filósofos naturales puede ser admitida también a los filósofos de la moral: los primeros suelen considerar muy frecuentemente un movimiento como compuesto de dos partes separadas entre sí, a pesar de que al mismo tiempo reconozcan que ese movimiento es en sí mismo simple e inseparable” (Hume 2005, 663).
} 
Podemos en este momento recapitular los dos efectos que se ciernen sobre la imaginación: por un lado, la imaginación sufre una inclinación (penchant) que es donada por la pasión, esta afección produce una relación real en la imaginación (paso desde lo posible a lo real). Este es el efecto simple. Ahora, en un segundo efecto (el complejo), la relación real producida en la imaginación por la pasión, que es univoca también le llama Deleuze, es reflejada a partir de la asociación constituyéndose en regla general "y valoriza lo más lejano, lo más distante" (Deleuze 1953, 59) yendo a un más allá de la inclinación de la imaginación: lo real se refleja. Este más allá (par-delà) debe pensarse como un dépassament, es decir, como un rebasamiento de la parcialidad en la cual estaba alojada la imaginación antes de este segundo efecto, el más complejo. Es decir, no solo se supera la parcialidad del espíritu con la fijación, sino que en un segundo momento, con el reflejo de la pasión, se va también más allá de la inclinación o el devenir naturaleza del espíritu. Deleuze también hablará de que a partir de los principios (la pasión y el entendimiento) se puede comprender cierta comprensión que tendría Hume sobre la naturaleza y la cultura, es decir, a partir de este efecto complejo se está realmente en el ingreso del sujeto a la cultura, o más bien, de la verdadera idea de subjetividad que el texto de Hume impone. Dicho de otra manera, cuando el espíritu es afectado por los principios de la asociación se está en presencia de cierto sujeto en un estado actual: la colección de ideas se asocia, deviene facultad humana. Sin embargo, dirá Deleuze, la idea de subjetividad ocurre realmente cuando la pasión es reflejada en la imaginación: una impresión de reflexión, resultado de una cualificación del espíritu: "lo que constituye el yo es, de hecho y ahora, la síntesis de la afección misma y de su reflexión, la síntesis de una afección que fija a la imaginación y de una imaginación que refleja a la afección" (Deleuze 1953, 59). En otros términos, si el principio de asociación vincula a las ideas, ésta asociación depende finalmente más que en sí misma en cierto afuera que Hume nomina como la circunstancia, o al decir de José Luis Pardo, "las variables históricas, política, económicas, etcétera, que explican que inventemos precisamente estas reglas e instituciones y poseamos precisamente estas creencias" (Pardo 2014, 31). En este mismo sentido es que Deleuze al comienzo de ES llama a Hume un sociólogo, un historiador, pero de un tinte bastante particular, ya que está concibiendo a Hume en una oposición radical contra la psicología, o lo que llama dentro de esa obra, una psicología del espíritu. Ser un sociólogo o un historiador, o un moralista, tiene relación con el principio fundamental que significa la pasión. Es en este sentido que debemos señalar que el empirismo de Hume está cruzado por cierta sensibilidad, o cierta afectividad, con la cual el sujeto se constituye realmente y se establece en su formación gradual. Sin cierta pasión no existe génesis de la subjetividad, y con ello tampoco ningún tipo de práctica. 


\section{CONCLUSIÓN}

¿Cuál sería la relación del empirismo, en este contexto, en $E S$, y lo que llamará empirismo trascendental en $D R$ ? De manera más frontal, lo trascendental tiene un tono afirmativo en el contexto de la tesis doctoral de nuestro autor. Ya que anteriormente lo presenta de manera negativa, como por ejemplo en $N P$, donde se estima solapadamente una cierta idea de lo trascendental, que se vislumbra por ejemplo, en lo que representa la genealogía o la crítica nietzscheana. Si bien se antepone de manera explícita a la filosofía kantiana, se verá que la voluntad de poder irrumpe como un principio trascendental, aunque sea omitida bajo este apelativo directo, pues se advierte más bien que la voluntad de poder se constituye como un principio que obedece a una génesis interna (Deleuze 1962, 104). Es que es en la lectura de Nietzsche donde Deleuze pareciera encontrar una torsión de lo trascendental, que se ampliará en el ensayo sobre Kant dos años más tarde, en donde se establece cierta concepción de la génesis en la estética kantiana a partir de los encuadres de Salomon Maimon. Sin embargo, este texto, aunque instala las directrices de una génesis trascendental en los términos de Deleuze, será profundizado en $D R$ a partir de dos lugares. El primero es la oposición descrita entre el condicionamiento y lo genético, pues arguye que el mero condicionamiento de la experiencia nos deja atrapados en los límites de una experiencia posible, y no en una experiencia real, cuestiones que en Le Bergsonisme están totalmente definidas, pero también en sus textos dedicados a Bergson en los años 50. En segundo lugar, la formulación de lo trascendental pasa por el problema de las facultades, o en términos más específicos, sobre la génesis de lo sensible. Debemos recordar que las facultades para Kant poseen cierto ordenamiento, que corresponde a concebirlas como dadas o presupuestas, sin estimar en una germinación o génesis de las mismas. Movimiento análogo a cuando en $E S$ Deleuze diga que la imaginación debe devenir facultad (p.3) a partir de un rebasamiento. Creemos, a partir de esto, que deslindar tan fácilmente un empirismo de un empirismo trascendental, dentro de los primeros años de trabajo deleuzeano, es un poco forzado. Nos parece que existe un arco, una trayectoria, con respecto al problema del empirismo en el corpus deleuzeano, que de manera gradual germina a partir de firmas y problemas que comparecen en la dirección de producir una metafísica singular. Los planteamientos de $E S$ nos parecen antecedentes más que gravitantes en lo que en $D R$ llamará empirismo trascendental, por sobre todo en cómo aparece la noción de génesis para explicitar el surgimiento de la subjetividad, pero también de la imaginación en tanto facultad. 


\section{BIBLIOGRAFÍA}

Canavera, Julien. 2012. "Hume en Deleuze. Los primeros lineamientos del empirismo trascendental" en Logos. Anales del seminario de metafísica. Vol. 45:123-144

Deleuze, Gilles. 1953. Empirisme et subjetivité. Essai sur la nature humain selon Hume. [ES] Paris: Presses universitaires de France (PUF). (Deleuze, Gilles. 2002. Empirismo y subjetividad. Traducción Hugo Acevedo (2da edición). Barcelona: Gedisa.)

Deleuze, Gilles. 1962. Nietzsche et la philosophie. [NP] Paris: PUF. (Deleuze, Giles. 1971. Nietzsche y la Filosofía. Traducción de Carmen Artal. Barcelona: Anagrama.)

Deleuze, Gilles. 1963. La philosophie critique de Kant. [PCK] Paris: Presses universitaires de France (PUF). (Deleuze, Gilles. 1997. La filosofía crítica de Kant. Traducción de Marco Aurelio Galmarini. Madrid: Cátedra.)

Deleuze, Gilles. 1968. Différence et répétition. [DR] Paris: Presses universitaires de France (PUF). (Deleuze, Gilles. 2002. Diferencia y repetición. Traducción de María Silvia Delpy y Hugo Beccacece. Buenos Aires: Amorrortu editores.

Deleuze, Gilles. 1983. L’image-mouvement. Cinéma 1. [IM] Paris: Editions de minuit. (Deleuze, Gilles. 1985. La imagen-movimiento. Estudios sobre cine 1. Traducción Irene Agoff. Barcelona. Paidós.)

Deleuze, Gilles. 2002. "Hume" en L'île déserte et autres textes (Textes et entretiens 1953-1974). [ID] Édition préparée par David Lapoujade, 226-237. Paris: Editions de minuit. (Deleuze, Gilles. "Hume" en La isla desierta y otros textos (Textos y entrevistas 1953-1974). Traducción de José Luis Pardo. Valencia: Pre-textos.)

Deleuze, Gilles. 2003. "Réponse à une question sur le sujet" en Deux regimes de fous (Textes et entretiens 1975 - 1995). Édition préparée par David Lapoujade, 326-328. Paris: Editions de minuit. (Deleuze, Gilles. 2008. "Respuesta a una pregunta sobre el sujeto" en Dos regímenes de locos (Textos y entrevistas 1975 - 1995). Traducción José Luis Pardo, 313-316. Valencia: Pre-textos.)

Deleuze, Gilles — Guattari, Félix. 1972. L’Anti-OEdipe. Capitalisme et schizophrénie. [AOE] Paris: Editions de minuit. (Deleuze, Gilles y Guattari, Félix. 1985. El Anti-Edipo. Capitalismo y esquizofrenia. Traducción Francisco Monje. Barcelona: Paidós).

Feuerhake, Ernesto. 2014. Empirismo y crítica. Husserl y Deleuze a través de Hume. Tesis de magíster Universidad de Chile. Santiago: Universidad de Chile.

Hume, David. 2005. Tratado de la naturaleza humana. Edición a cargo de Félix Duque (4ta edición). Madrid: Tecnos.

Lapoujade, David. 2016. Deleuze. Los movimientos aberrantes. Traducción Pablo Ires. Buenos Aires: Cactus.

Martelaere, Patricia. 1984. "Gilles Deleuze, interprete de Hume” en Revue Philosophique Louvain, Quatrième série, tome 82, n54, pp. 224-248. 
Montenegro, Gonzalo. 2013. Empirismo trascendental. Génesis y desarrollo de la filosofía de Gilles Deleuze. Tesis doctorado Universidad de Chile. Santiago: Universidad de Chile.

Pardo, José Luis. 2014. A propósito de Deleuze. Valencia: Pre-textos. 